\title{
USING TRANSIENT SIMULATIONS TO IMPROVE FIELD SERVICE SYSTEMS FOR SEMICONDUCTOR MANUFACTURING
}

\author{
Dr. John W. Fowler \\ Arizona State University \\ P.O. Box 874706 \\ Tempe, Arizona 85287 USA
}

\author{
Dr. Esma S. Gel \\ Arizona State University \\ P.O. Box 875906 \\ Tempe, Arizona 85287 USA
}

\author{
Dr. Cem Vardar \\ Arizona State University \\ P.O. Box 875906 \\ cem.vardar@asu.edu
}

\begin{abstract}
Simulation models of complex real world systems require long computation times for producing reliable estimates of the system performance measures of interest. However, it has been observed in the simulation-based optimization literature that the order and ranking relationship between solutions often become significant very early in the simulation runs, even during the transient state. In this paper we propose a method for using very short transient simulation runs to compare different alternatives which can be quite useful in metaheuristics such as genetic algorithms. We present initial experimentation results for a simple problem system $(\mathrm{M} / \mathrm{M} / 1)$ and a field service system design problem from semiconductor industry.
\end{abstract}

Keywords: Transient Simulation, Simulation-based Optimization, Heuristic

\section{INTRODUCTION}

Analysis of large real world systems is a difficult task due to the complexities that arise when there is randomness inherent in the system. However, some degree of randomness is a common and unavoidable characteristic among almost all real-world systems. Simulation modelling has been widely used as a descriptive analysis tool to obtain performance measure estimates of such systems under given system configurations.

Although computer simulation modelling is a highly effective tool for descriptive modelling, analysts often need to search for model decision parameters that either maximize or minimize one or more performance measures of the system. This type of a problem is referred to as a simulation optimization problem. Fu (2001) formally defines a simulation-based optimization problem as finding a configuration or a design that minimizes the objective function:

$$
\min _{\theta \in \Theta} J(\theta)=E[L(\theta, \omega)]
$$

where $\theta$ represents the (vector of) decision variables, $J(\theta)$ is the objective function, $\omega$ represents a sample path (simulation replication), and $\mathrm{L}(\theta, \omega)$ is the sample performance measure estimate.

The difficulty of solving this problem is that $\mathrm{J}(\theta)$ is an implicit function of the decision variables (i.e., $\theta$ ) and an observation of $\mathrm{J}(\theta)$ can only be obtained through an execution of the simulation model. Often, this requires long computation times, particularly when initial transient bias is significant. Particularly in the semiconductor industry, simulation models used for various purposes, such as 
solving supply chain and wafer fab operation problems, are generally quite large and require long computation times to obtain reliable estimates of the performance measures of interest.

Since simulation modelling is traditionally a descriptive analysis tool, in practice, simulation execution parameters such as the level of detail in the model, run length, number of replications, and warm up period are usually set to achieve a sufficiently accurate and precise evaluation of the given system configuration. Most simulation optimization techniques proposed in the literature accept these parameter settings as given and build upon this assumption. That is, they assume that an execution of a simulation model $\mathrm{L}(\theta, \omega)$ provides an unbiased estimate for $\mathrm{J}(\theta)$. Observe that this assumption is inherent in the formal definition of the simulation optimization problem. However, getting an unbiased estimate of $\mathbf{J}(\theta)$ typically requires long computation times especially when the variability inherent in the system is significant, since the accuracy of the confidence interval around $J(\theta)$ cannot improve faster than $1 / \sqrt{ } \mathrm{N}$. In addition the existence of a transient bias for most real world system adds significant computation burden for estimating the steady state performance.

In this paper, we investigate the use of short transient simulation runs to compare solution alternatives in the presence of design-dependent bias and estimation noise. We propose a method that improves the efficiency of using short transient simulations to estimate the ranking of the solution alternatives. Our method uses data collected during the simulation run at different time intervals to predict design-dependent estimation error and ranking of solutions. We demonstrate our method using experimental results on an $\mathrm{M} / \mathrm{M} / 1$ queueing system and a problem from semiconductor manufacturing that deals with designing a maintenance service system for wafer fabrication facilities.

\section{RELEVANT LITERATURE}

Our approach is motivated by the seminal paper, Ho et al. (1992), which proposes a concept called 'ordinal optimization'. Ordinal optimization finds a good, better or best solution, instead of trying to accurately estimate the performance of the systems. Ordinal optimization can be complementary to the current simulation optimization techniques like the one proposed in Boesel et al. (2003) by reducing the massive search space into a manageable size before applying sophisticated performance evaluation techniques.

Ho et al. (1992) demonstrate that the order relation between systems often becomes significant very early in the simulation runs and without making many replications. In their experiments, they show that promising solutions could be differentiated from inferior solutions with very little simulation computing time.

The research that followed Ho et al. (1992) can be generally classified in two threads: applications of ordinal optimization ideas and theory of ordinal optimization ideas. Ganz and Wang (1994), Ho and Larson (1995), Wieseltier et al. (1995), and Yang et al. (1997) apply ordinal optimization ideas to various real world problems. On the theory side, Dai (1996) and Xie (1997) prove that the convergence rate of order of two systems can be exponential as the simulation effort expanded increases. Lau and Ho (1997) formalize the idea of ordinal optimization with the definition of alignment probability, which represents the probability of having a specified number of "good enough" solutions in a selected subset of the design space. The authors define the concept and tabulate alignment probabilities for the horse race selection rule for different forms of order performance curves. The basic assumption is that the noise in the observations does not depend on the alternative.

Yang et al. (1997) investigates various options when this important assumption is violated. They propose a method for estimating the design-dependent noise by a linear regression approach. They use this method in two real world examples and demonstrate improvements over traditional ordinal optimization techniques. Yang and Lee (2002) relaxes the assumption of design-dependent noise and propose methods of selection when the noise is design-dependent but known.

In the ordinal optimization literature, short simulations are one of the methods used for selecting a set of good enough alternatives using a fixed simulation run length which has to be specified beforehand. To best of our knowledge there has been no study about how to choose and/or adapt the simulation run length for making the comparisons. In this paper we propose a heuristic which uses variable run lengths to compare the alternatives based on the information that can be obtained from the initial observations in the simulation. In addition we consider comparison of only two alternatives with respect to each other rather than a selection of a subset of good enough alternatives from a large 
set. This case is particularly important for metaheuristics used in commercial simulation optimization packages where comparison of two solutions has to be made repetitively.

In the traditional simulation literature there is a vast amount of research papers which mainly study how to deal with the initial transient bias for estimating steady state mean. A comprehensive review of the methods for dealing with transient bias can be found in Law (2014) and progress is still being made. The main focus of most of these studies is how to remove and/or minimize the effects of transient stages of a simulation run where the main objective is to estimate steady state performance rather than utilizing the information generated during the transient stages of the simulation. However, in some recent studies the idea of using transient simulation data to draw inferences about steady state behavior is being explored. For example, Voss et al. (2005) utilizes maximum likelihood estimators for the mean of an autoregressive process to construct confidence intervals for steady state mean from transient simulation data. Although the method proposed is asymptotically valid for linear autoregressive series, they do not work well for models where the initial transient bias is significant such as an M/M/1 queueing system loaded over 50\% utilization. For realistic systems the order of autoregression has to be also estimated by using the highly variable transient data which results in poor coverage for the confidence intervals constructed. Our paper differs from this study by focusing mainly on the ranking of two different alternatives using transient data rather than estimating their respective steady state performance.

\section{PROPOSED METHOD}

The idea of using short transient simulation runs to compare two alternatives is based on the observation that ranking relationship between systems usually becomes significant much before the systems reach their respective steady states in a simulation run (Ho et al. 1992). That is, if we only need the ranking between two different alternatives and are not interested in estimating the steady state performance of the systems we can use much shorter simulation runs for making the correct comparison. Estimating the ranking with a short computation time rather than spending the computation budget on estimating the steady state performance could particularly be useful in simulation-based optimization techniques that utilize metaheuristics such as genetic algorithms and simulated annealing.

Based on this observation, a method that could be applied is estimating the ranking between different alternatives based on their observed transient performance. The observed transient ranking of two alternative systems from a single simulation run might not reflect the true steady state ranking due to two reasons. The first one is the inherent simulation variability which might be rather high in short transient simulations due to correlated observations and small sample size. We have some degree of control on this type of error through the length and number of replications we use for comparing the system alternatives. The second reason is true average transient ranking of two systems might not reflect the true steady state ranking relationship. That is, when comparing two alternative systems, the alternative with the worse steady state performance might have a better expected transient performance. The usefulness and efficiency of this method depends on the transient behavior ranking relationship between the two systems to be compared. Below, we define three groups of systems for categorizing this relationship.

1) Dominating ranking relationship: The average transient ranking between two systems reflects the true steady state ranking of the systems at any simulation length. Comparison of two systems with a dominating relationship can be made at any simulation length without making a systematic error. That is at each simulation length the true average transient ranking reflects the true steady state ranking. When comparing these systems with this type of ranking relationship based on a single replication we can make an incorrect selection only due to simulation variability.

For example, consider following two M/M/1 system alternatives

Alternative 1: $\rho=0.75$ with $\lambda=0.5$ and $\mu=0.667$

Alternative $2: \rho=0.80$ with $\lambda=0.6$ and $\mu=0.75$

Figure 1 shows the average time in system for both alternatives based on the number of customers simulated after starting in an empty and idle state. Observe that, if we want to select the system with lower time in system by simulating two systems starting in an empty and idle state, we can use the 
transient observations of time in system with any simulation length because alternative 1 has a lower expected time in system than alternative 2 even in the transient stages starting with in an empty and idle state.

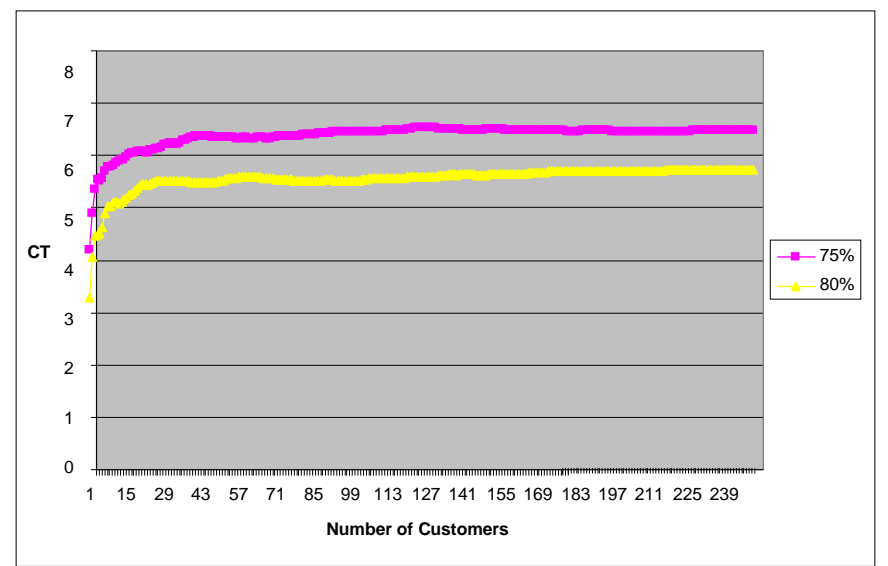

Figure 1 Average time in system for two alternatives with dominating ranking relationship

Problems with alternatives that have a dominating transient ranking relationship are the best candidates for using short transient simulations for estimating steady state ranking.

2) Single crossover ranking relationship: There is a cutoff simulation length point after which the observed ranking between two systems reflects the steady state ranking of the systems and before which the observed ranking is the reverse of the steady state ranking.

For example consider following $\mathrm{M} / \mathrm{M} / 1$ systems:

Alternative 1: $\rho=0.65$ with $\lambda=0.250$ and $\mu=0.384$

Alternative 2: $\rho=0.85$ with $\lambda=0.497$ and $\mu=0.584$

Figure 2 shows the average time in system for both alternatives based on number of customers simulated after starting in an empty and idle state. If we want to select the system with lower time in system by simulating two systems starting in an empty and idle state and make the decision based a simulation of the first 10 customers starting in an empty and idle state we would be making a systematic error. Although alternative 1 has a larger average time in system in initial phases of the transient state, it has a higher average time in system than alternative 2 in steady state. For these types of systems, only the observations after the crossover point should be used for estimating the steady state ranking.

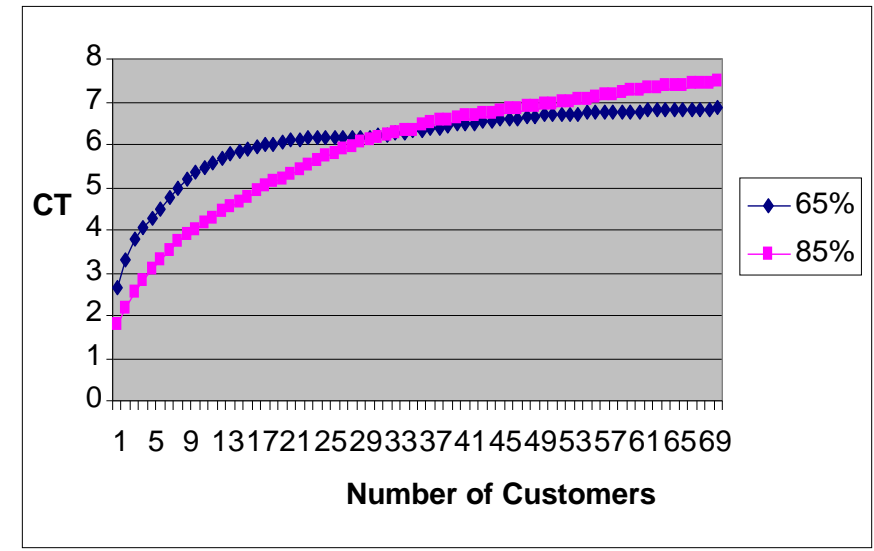

Figure 2 Two alternatives with single crossover ranking relationship

Problems where different solution alternatives that have a single crossover relationship can still be good candidates for applying this method if the crossover point is very early in the transient stage or can be accurately predicted. This type of relationship is often observed when comparing two alternatives for real world problems. 
3) Multiple crossover ranking relationship: The ranking relationship in the transient state between alternatives changes multiple times until the systems reach at steady state. These types of systems are not good candidates for using transient simulations. Queueing systems with non-trivial batching policies or systems that include periodic queueing elements potentially belong to this group.

For alternatives that have a dominating ranking relationship any ranking observation made during the transient period can be expected to give a correct inference about the steady state ranking relationship of the alternatives. An incorrect ranking can be only observed due to inherent simulation randomness. In a similar fashion, for alternatives that have a single crossover relationship only the ranking observations made after the crossover point can be expected to give correct inference. However, in metaheuristics such as genetic algorithms, incorrect decision in the exploration (early) phase are not overly harmful.

A difficulty that needs to be considered for using transient simulations for estimating the steady state ranking of two systems is the high variability in the transient solutions due to a low number of observations. Even for alternatives that have a dominating or single crossover ranking relationship the comparison decisions that are made based on initial observations may not be reliable due to the fact that comparisons are made based on a single replication. A method that uses transient simulation runs for comparing alternatives should include a mechanism for controlling high variability in the transient observations. In steady state simulations with independent replications (or independent batch means) confidence intervals are used to control the level of error in the inferences drawn from simulation results. Since observations in short transient simulations are highly correlated, straightforward confidence interval methods cannot be applied exactly. However, in the following sections of this paper we demonstrate that confidence intervals that assume independence of observations could be useful for approximately controlling the error. We demonstrate in Vardar (2006) that using common random numbers in different alternatives further improve the control of error.

\subsection{Variable length transient ranking heuristic}

In this section, we define the proposed heuristic. The basic idea of our heuristic relies on making short simulations of two systems to be compared, and comparing the performance difference between the two systems at different run lengths. At each comparison point we calculate a statistic using the observations made until that point. Based on this statistic, we either decide to take additional observations from both systems or stop and make a decision on the ranking of the alternatives based on the observations obtained so far. This type of dynamic data collection from simulation is common in the ranking and selection literature but it has mainly been used for steady state simulations (Picthitlamken et al., 2006).

We take observations in batches of $\mathrm{k}$ from both systems that will be compared. $\mathrm{k}$ could be in terms of numbers of arrivals to the system or in terms of simulated time. $\bar{x}_{i}^{n}$ is the average of all the observations taken from system $i$ in the $n t h$ batch. $\bar{X}_{i}^{n}$ is the cumulative average of alternative $i$ after the $n t h$ batch. We denote the difference between the systems in batch $n$ with $\bar{y}^{n}\left(\bar{y}^{n}=\bar{x}_{i}^{n}-\bar{x}_{j}^{n}\right)$ and the cumulative difference between the systems after batch $n$ with $\bar{Y}^{n}\left(\bar{Y}^{n}=\bar{X}_{i}^{n}-\bar{X}_{j}^{n}\right)$. We start with taking 3 batches of observations from each alternative and construct a pseudo-confidence interval with half length $(H L)$ width using the following formula.

$$
H L=t_{1-\alpha / 2, n-1}\left(\frac{s_{n}}{\sqrt{n}}\right)
$$

Here $t_{1-\alpha / 2, n-1}$ is the upper critical value for $1-\alpha / 2$ critical value for the $t$ distribution with $n-1$ degrees of freedom. $s_{n}$ is the sample standard deviation of the batch average differences $\left(\bar{y}^{1}, \bar{y}^{2}, \ldots, \bar{y}^{n}\right)$ calculated using the formula.

$$
s_{n}{ }^{2}=\frac{\sum_{t=1}^{n}\left(\bar{y}^{n}-\bar{Y}^{n}\right)^{2}}{n-1}
$$


If $\left|\bar{Y}^{n}\right|>\frac{H L}{r}$ then we stop and make a decision on the ranking of two alternatives based on their current observed performance. In this formula $r$ is a scaling factor to control the average run length used for making the comparisons. If this inequality is not satisfied we take one additional batch from each alternative and calculate the updated $H L$ and compare it with the updated cumulative difference. We continue to iterate until a ranking decision is made.

The pseudo code for our heuristic can be seen below.

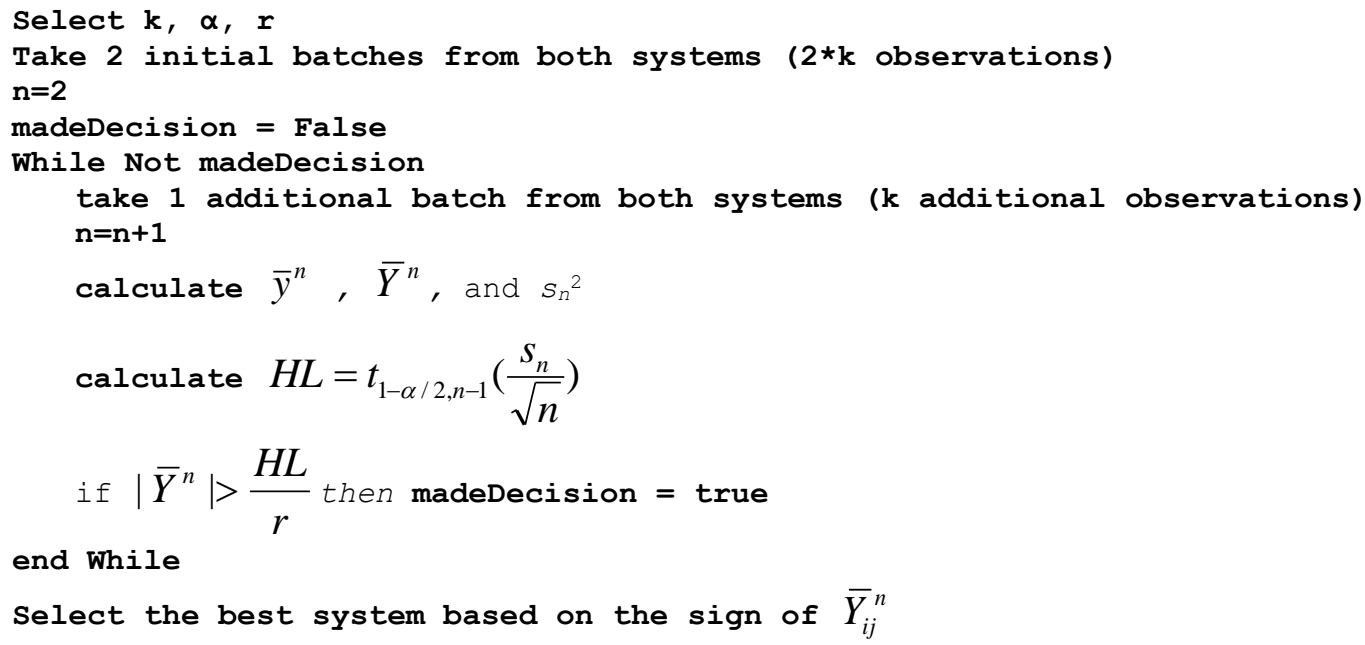

Statistic $H L$ is calculated using the formula for the half length of a confidence interval with $\alpha$ level of confidence with $n$ independent observations. Since we are using very small batch sizes and collecting data during the transient period observations are correlated. This often results in the underestimation of $s_{n+}$ and invalid confidence interval half lengths. However, this is not a major problem since our objective is to compare performance of two alternatives rather than estimating a precise confidence interval of the performance difference.

We also use a scaling factor, $r$, to control the average run length used for making the comparisons. When we pick a small $r$ more evidence is required for making a comparison which results in longer runs on the average. Whereas when $r$ is big comparison decision can be made with shorter runs. As expected longer runs result in higher probability of correct selection. Thus, $r$ should be chosen based on the correct selection requirements of the application and the available computational budget.

\section{COMPARISON OF VARIABLE AND FIXED RUN LENGTH FOR THE M/M/1 SYSTEM}

We use the M/M/1 queueing problem used in Yang and Lee (2002) as one of our test problems. In this problem, we would like to find the $\mathrm{M} / \mathrm{M} / 1$ system that gives the minimum average cycle time from a set of 1,000 different alternatives. The alternatives are determined by varying the arrival rate $\lambda$ from 0.01 to 1.01 in steps of 0.001 and the service rate $\mu$ changes accordingly to keep the utilization of the system constant at $\rho$. Note that, in this setting we know the optimal alternative: $\lambda=1.01$ is the best possible configuration, since it minimizes cycle time. (Recall that cycle time is equal to $1 /(\mu-\lambda)$ in an $\mathrm{M} / \mathrm{M} / 1$ system.) We consider $\rho=0.65$ and 0.95 as two different versions of this problem.

Our objective in the experimentation is to estimate how well our heuristic performs when comparing two solutions picked randomly from the different alternatives in the solution space. Metaheuristics such as genetic algorithms and simulated annealing are widely used in commercial simulation optimization packages and require the implementation of repetitive pairwise comparisons between solutions during the search for an optimal solution.

Although this is a very simple system, transient bias particularly for higher $\rho$ values is hard to eliminate. For example, for the test problem with $\rho=0.8$ and $\lambda=0.5$, we might need to run the simulation until 10,000 customers are served before we start to get a reliable estimate of the cycle 


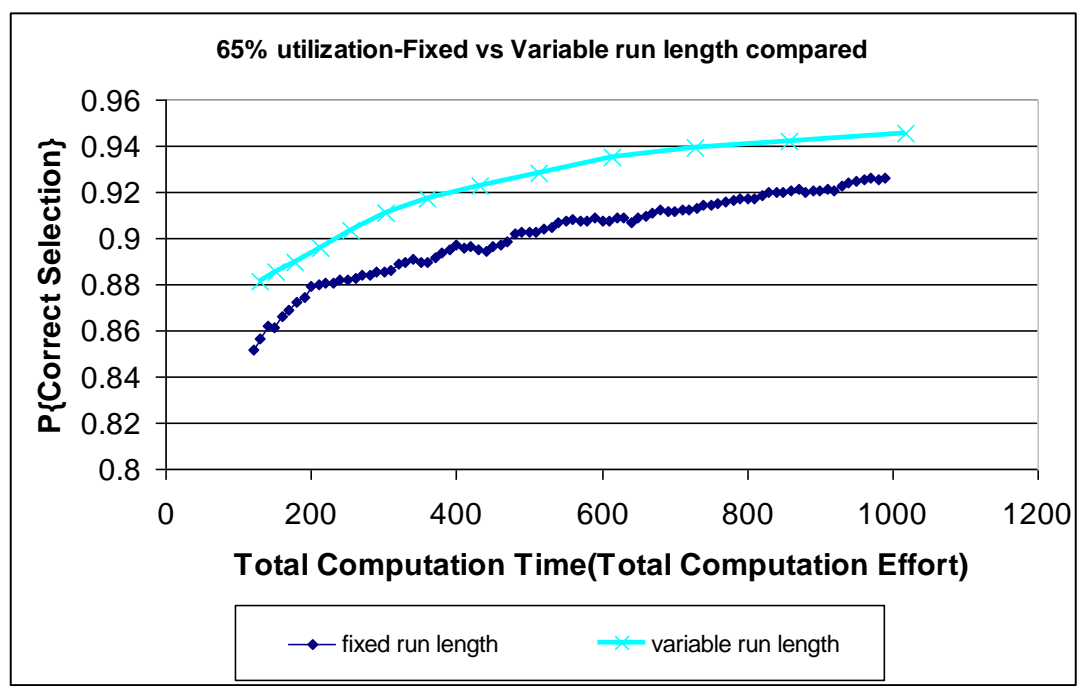

Figure 3 Fixed run length vs. variable run length simulation at $65 \%$ utilization of $M / M / 1$ system

time. Whereas, when comparing two solutions, we can achieve probability of correct selection values as high as $80-90 \%$ with just 100 customers (i.e., 1/100th of the time required to get a reliable estimate of cycle time). Metaheuristics used in commercial simulation optimization software are fairly robust to this level of noise (Boesel et al., 2003).

We now present results from experiments that we have conducted using two different methods that use transient simulation data to compare two alternatives. The first method is the fixed run-length method in which we take a prespecified number of observations from the system and make the decision based on the observed performance of the two systems that are compared. This corresponds to the traditional ordinal optimization approach where the simulation run length specified beforehand. The second method is the variable run length heuristic described in section 3 . The variable run length heuristic compares the statistic calculated based on the observations obtained so far with the cumulative difference between the two alternatives. If the absolute value of the statistic is greater than the absolute value of the difference between two alternatives, the comparison is made based on the observed performance at that point. Otherwise, more observations are taken from the simulation.

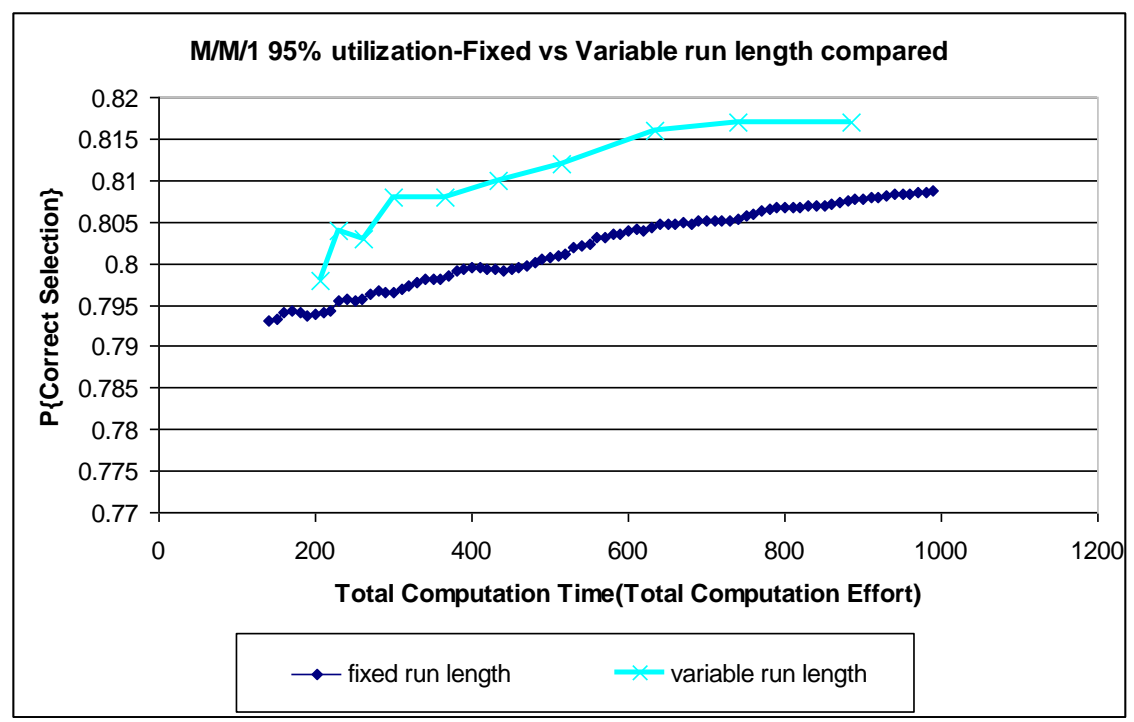

Figure 4 Fixed run length vs. variable run length simulation at 95\% utilization of M/M/1 system

In the experimentation, we pick two solutions at random from the solution space $(1,001$ solutions). For the fixed run length method, we make a new comparison each time 10 additional customers are served and record the success rate of correctly identifying the better solutions. This way we cover the complete range of possible run lengths that can be used with the fixed run length 
method. For the variable run length method, we use a batch size of $10(\mathrm{k}=10)$ and a $95 \%$ confidence level for the pseudo confidence interval. We change the run time coefficient parameter, $r$, between 0.1 and 1.4 to generate similar total computational run time to the fixed run length method. We pick 1,000 solution pairs and perform 30 replications for each pair. The following graphs (Figures 3 and 4) show the estimated probability of correct selection for two different utilization cases $\rho=.65$ and $\rho=.95$. The computation effort on the $\mathrm{x}$-axis is number of customers.

For the same computation effort variable run length method has a higher probability of correct selection for all utilization and all computation times. The improvement of the variable run length heuristic could be seen better if average run length required for the same probability of correct selection is considered. For example to achieve a $90 \%$ correct selection for $\rho=.65$ with the fixed run method requires around 400 customers on the average whereas for the variable run length heuristic, the same probability of correct selection can be achieved with 200 customers - around 50\% reduction in the total computational time. Furthermore, the run time improvements are much higher as the utilization increases.

\section{FIELD SERVICE SYSTEM LOCATION AND CAPACITY PROBLEM}

Our second problem deals with strategic field service planning in the semiconductor manufacturing industry. In this problem, a field service provider has to decide the location and type of the regional service centers to open and the number of service engineers (of different types) to minimize the total fixed regional service center opening, personnel, travel, technology can contractual penalty costs. The problem is further complicated with capability of using a technology called remote diagnostics, which enables the service provider to respond to some portion of service requests remotely. We investigate an instance of this problem with seven customers to serve, four possible regional service center locations, two different service engineer types and two different types of regional service centers (as shown in Figure 5). The nodes in the figure represent the queueing network of service engineers handling service requests from fabs. Each solution represents a possible alternative for the system with respect to which service centers are opened, how many service engineers are employed at each location at each level, and the assignments of fabs to service centers. The objective is to determine the solution that minimizes the expected total cost of the field service provider. Due to second order congestion effects, the expected cost of different solution alternatives can only be estimated using a simulation model. This system is a very complicated system with high variability. To reach steady state and get a reliable estimate for the total expected cost, 10,000 to 20,000 hours of operation has to be simulated. A more detailed description of the problem can be found in Vardar et al. (2007).

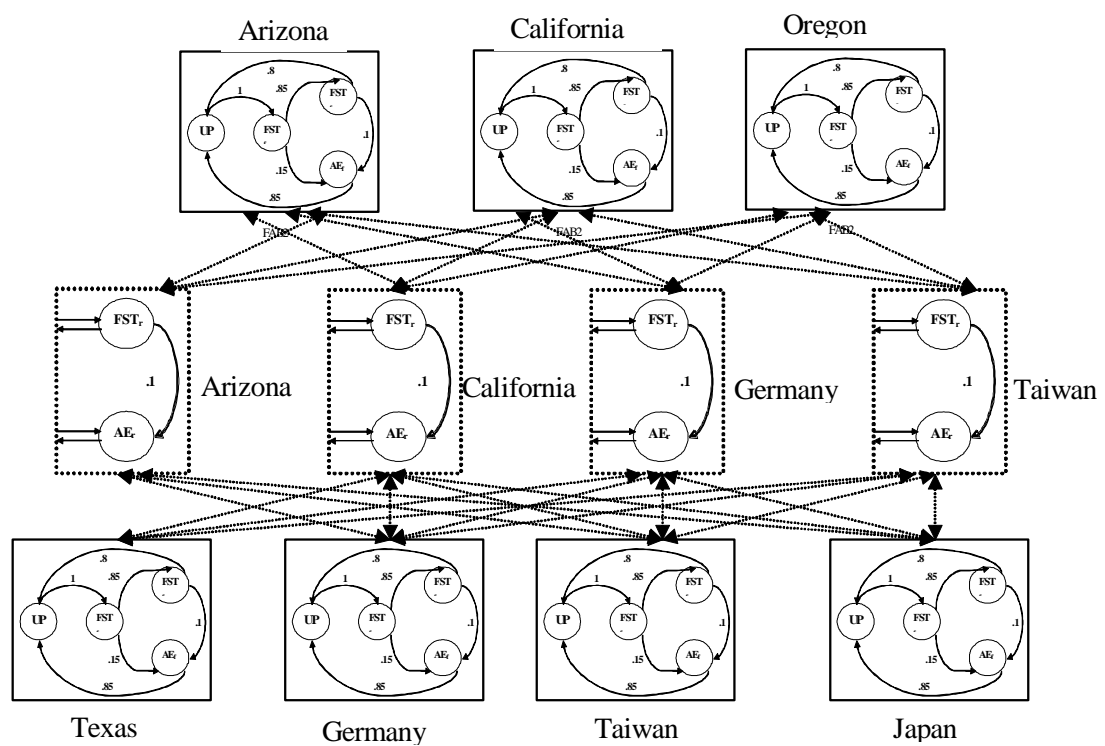

Figure 5 Field service system design problem
Fabs

Service provider's regional locations Fabs 
We now present results from initial experiments that we have conducted with two methods that use transient simulation data to compare two solution alternatives for the field service system design problem. We, again, compare the fixed run length method and variable length heuristic described above. For the variable run length method, we use a batch size of 96 hours $(\mathrm{k}=96 \mathrm{hours})$ and a 95\% confidence level for the pseudo confidence interval. We change the run time coefficient parameter, $r$, between 0.1 and 1.4 to generate similar total computational run time to the fixed run length method. In the experimentation, we pick two solutions at random from the solution space of 1,000 random solutions and make 30 replications for each pairwise comparison. We note that the results are similar to the results in the $\mathrm{M} / \mathrm{M} / 1$ experiments.

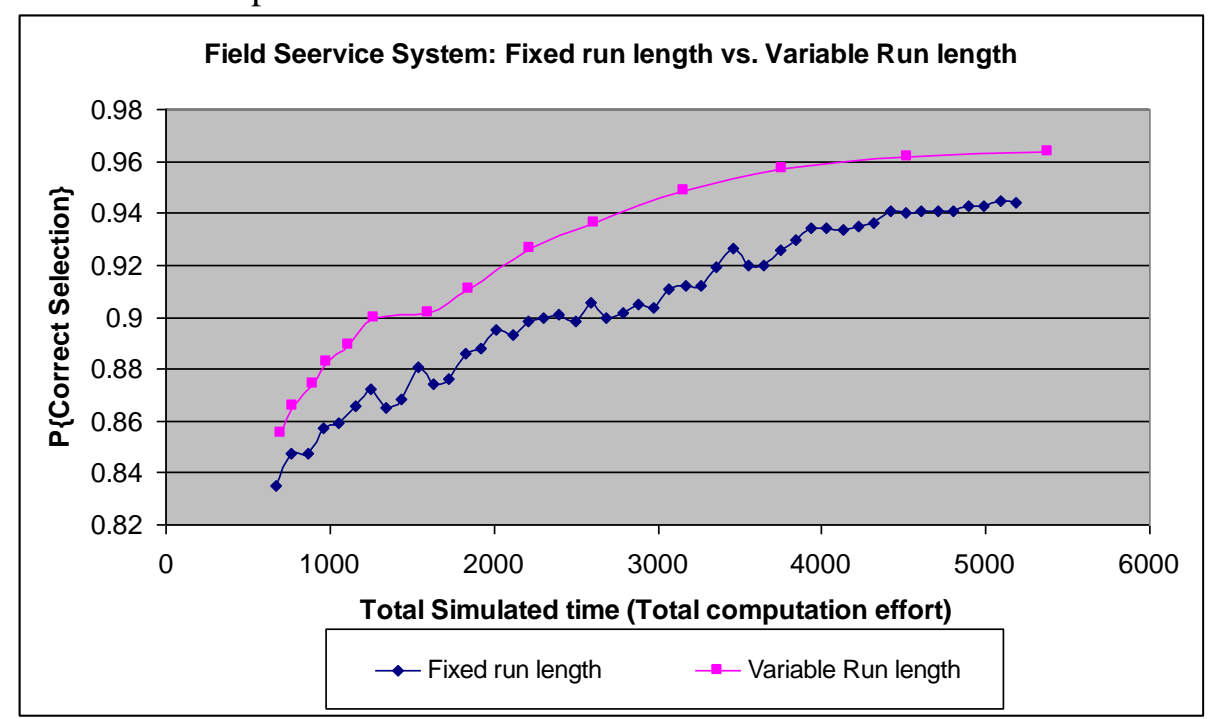

Figure 6 Fixed and variable run length heuristic compared for the field service design problem

\section{CONCLUSIONS AND DISCUSSION}

In this paper, we have presented a new method for using transient simulation runs to compare different solution alternatives for simulation-based optimization. Although transient simulation runs do not provide good estimates for the performance of alternatives, they can provide fairly consistent and useful inferences about how an alternative performs compared to another alternative. This type of inference is particularly useful in metaheuristics commonly used in commercial simulation-based optimization packages where this type of comparison between alternatives has to be made repetitively. In some cases, adequate correct selection rate can be obtained from transient simulation using $1 / 100$ th of run time needed for reaching steady state.

We presented initial experimentation results for two different problems: an $M / M / 1$ system and a field service system design problem from the semiconductor industry. Our dynamic method provides the same level of correct selection with significantly less simulation run time in all cases presented. The threshold and step size parameters used in our method have an effect on the performance of the algorithm. Vardar (2006) explores ways to find effective values of the parameters in our method, independent of the problem type. It also modifies the variable run length heuristic to consider whether or not the sample paths of the transient solutions of the two solutions are trend converging or diverging which is helpful, particularly in the case of the single crossover ranking relationship.

Until recently, simulation-based optimization was not a practical tool to be used in problems that are faced by the semiconductor industry, since these problems often required the use of complex simulation models and long run times. However, advanced metaheuristics and the use of ordinal optimization ideas can open the way for successful use of simulation-based optimization in semiconductor industry for operational and strategic problems.

\section{REFERENCES}

Boesel J, Nelson B L, and Kim S H (2003). Using ranking and selection to 'clean-up' after simulation optimization. Operations Research 51: 814-825. 
Dai L Y, (1996). Convergence properties of ordinal comparison in the simulation of discrete event dynamic systems. Journal of Optimization Theory and Applications 91(2): 363-388.

Fu M C (2001). Optimization for simulation: theory and practice. INFORMS Journal on Computing 14 (3): 192-215

Ganz A and Wang X (1994). Efficient algorithm for virtual topology design in multihop lightwave networks. IEEE/ACM Transactions on Networking 2(3): 217-225.

Ho Y C, Sreenivas R S, and Vakili P (1992). Ordinal optimization of DEDS. Discrete Event Systems: Theory and Applications 2: 61-88.

Ho Y C and Larson M E (1995). Ordinal optimization approach to rare event probability problems. Journal of Discrete Event Dynamic Systems 5(2-3): 281-301.

Lau T W E. and Ho Y C (1997). Universal alignment probability and subset selection for ordinal optimization. Journal of Optimization Theory and Applications 93(3): 455-489.

Law A (2014). Simulation modeling and analysis 5th edition. McGraw-Hill, New York.

Pichitlamken J, Nelson B L, and Hong L J (2005). A sequential procedure for neighbourhood selection-of-the-best in the optimization via simulation. European Journal of Operational Research 173: 283-298.

Vardar, C. (2006). A novel simulation optimization approach for a remote diagnostics capable field service system design problem. Arizona State University Dissertation.

Vardar C, Gel E S, and Fowler J W (2007). A framework for evaluating remote diagnostics investment decisions for semiconductor equipment suppliers", European Journal or Operational Research 180: 1411-1426.

Voss P A, Willemain T R, Haddock J, (2005). Estimating the steady-state mean from short transient simulation. European Journal of Operational Research 162, 403-417.

Wieseltheir J E, Barnhart C M, and Ephremides A (1995). Ordinal optimization of admission control in wireless multihop voice_data network via standard clock simulation. Journal of Discrete Event Dynamic Systems, 5: 243-280.

Xie X L (1997). Dynamics and convergence rate of ordinal comparison of stochastic discrete event systems. IEEE Transactions on Automatic Control, 42(4): 586-590.

Yang M S, Lee L H and Ho Y C (1997). On stochastic optimization and its applications to manufacturing. Lectures in Applied Mathematics, AMS-SIAM, 33: 317-331.

Yang M S, and Lee L H (2002). Ordinal optimization with subset selection rule. Journal of Optimization Theory and Applications 113(3): 597-620.

\section{AUTHOR BIOGRAPHIES}

JOHN FOWLER is the Motorola Professor of Supply Chain Management in the W.P. Carey School of Business at Arizona State University. His research interests include discrete event simulation, deterministic scheduling, and multi-criteria decision making. He has published more than 125 journal articles and more than 100 conference papers. https://isearch.asu.edu/profile/36406

ESMA GEL is an Associate Professor in the School of Computing, Informatics and Decision Systems Engineering in the Ira A. Fulton Schools of Engineering at Arizona State University. Her research focuses on the use of applied probability techniques for modelling, design and control of production systems and supply chains, with particular emphasis on workforce engineering. Her work has been published in leading journals and funded by the National Science Foundation, as well as industrial partners such as Intel, IBM and Infineon. Gel is a member of INFORMS, IIE and ASEE. https://isearch.asu.edu/profile/257696

CEM VARDAR is a Principal Optimization Engineer at Carvana. He designs, develops, tests and maintains decision support systems based on mathematical models that run on massive data sets. $\mathrm{He}$ approaches decision support system design from an engineering perspective and not merely as scientific problems. https://www.linkedin.com/in/cem-vardar-70119514/ 